\title{
Germanica
}

\section{«Immer noch Mutter / Mehr noch Mutter jetzt...» Brechts episches Spiel mit der Mutterimago}

"Toujours mère/Encore plus mère maintenant... "-Jeu épique brechtien avec l'imago maternelle

Ingrid Haag

\section{OpenEdition}

\section{Journals}

Édition électronique

URL : http://journals.openedition.org/germanica/1966

DOI : 10.4000/germanica. 1966

ISSN : 2107-0784

Éditeur

Université de Lille

Édition imprimée

Date de publication : 30 juin 1996

Pagination : $37-55$

ISBN : 9782098426320

ISSN : 0984-2632

Référence électronique

Ingrid Haag, « «Immer noch Mutter / Mehr noch Mutter jetzt...» Brechts episches Spiel mit der

Mutterimago », Germanica [Online], 18| 1996, Online erschienen am: 17 Juni 2013, abgerufen am 06

Oktober 2020. URL : http://journals.openedition.org/germanica/1966 ; DOI : https://doi.org/10.4000/

germanica.1966

Ce document a été généré automatiquement le 6 octobre 2020.

(c) Tous droits réservés 


\section{«Immer noch Mutter / Mehr noch Mutter jetzt...» Brechts episches Spiel mit der Mutterimago}

«Toujours mère/Encore plus mère maintenant... " - Jeu épique brechtien avec l'imago maternelle

Ingrid Haag

Alles übergab ich dem Staunen

Selbst das Vertrauteste

Daß die Mutter dem Kinde die Brust reichte.

Lied des Stücke Schreibers.

\section{Die Definition muß praktikabel sein}

Wir können den andern nur begreifen, wenn wir in ihn eingreifen können. Auch uns selbst können wir nur begreifen, indem wir in uns eingreifen ${ }^{1}$.

Sich und den andern in den Griff bekommen - die Herausforderung ist dort am stärksten, wo die Konfliktbewältigung am problematischsten erscheint: in der MutterSohn-Beziehung, von der Brechts Texte vornehmlich handeln ${ }^{2}$. Setzt das vom Stückeschreiber postulierte «eingreifende Denken»" Distanz voraus und Abgrenzung, so beschwört die Mutter-Sohn-Beziehung die Symbiose-Phantasie herauf, faszinierend und bedrohlich zugleich, zwischen dem Wunsch nach intimster Vereinigung und der Angst des Autonomieverlustes oszillierend. Der Mutterschoß - imaginierter Ort ersten Glücks-Versprechens und gleichzeitig traumatisches Bild für die Bedrohung des Verschlungenwerdens, vagina dentata:

Es war eine Lieb zwischen Füchsin und Hahn

"Oh, Goldener, liebst du mich auch?"

Und fein war der Abend, doch dann kam die Früh

Kam die Früh, kam die Früh:

All seine Federn, sie hängen im Strauch ${ }^{4}$. 
2 Irreduzible Ambivalenz, die bereits kennzeichnend ist für die frühen poetischen Szenarien der Ursprungsphantasien Brechts, genauer des «armen B.B.»:

Ich Bertolt Brecht, bin aus den schwarzen Wäldern.

Meine Mutter trug mich in die Städte hinein

Als ich in ihrem Leibe lag. Und die Kälte der Wälder

Wird in mir bis zu meinem Absterben sein ${ }^{5}$.

Die Bilder des Ursprungsorts (die schwarzen Wälder, der Mutterschoß), die sich um die Pole Kälte und Wärme artikulieren, sind eingespannt in ein Netz widersprüchlicher, nicht fixierbarer Bedeutungen, in denen sich das gespaltene Subjekt spiegelt, oder, wie Hans-Thies Lehmann formuliert, «lustvoll Identität aufs Spiel setzt» ${ }^{6}$. Auf der epischen Bühne nun setzt sich dieses Spiel fort, und zwar weiterhin sicherlich als lustvolles, wobei es uns allerdings scheint, daß die Mittel und Verfahren des Stückeschreibers immer mehr darauf abzielen, das Spiel in den Griff zu bekommen, das Risiko des Spieleinsatzes zu verringern; «aufs Spiel setzen» wohl noch, aber mit dem Gestus des souveränen Spielleiters. Wie hinte den gelungenen «Inszenierungen» der primäre Konflikt durchscheint, soll uns im folgenden interessieren?

In dieser Perspektive soll die Regression in die Dyade MutterSohn mit Peter von Matt als kreative ins Auge gefaßt werden: als der «produktive Rückgriff auf ein verfügbares Instrumentarium der Konfliktbewältigung» ${ }^{8}$. Eine der klassischen Strategien zur Beherrschung des Ambivalenzkonflikts, die Objektspaltung, hier die Mutterfigur betreffend, ist in Brechts Texten geradezu augenfällig9. Vor dem Hintergrund der Spaltung des Frauenbildes in Hure und Heilige, pendelnd zwischen Liebe und Haß, entfaltet sich die Phantasie von der bösen und der guten Mutter, der warmen und der kalten, der nährenden und der verschlingenden, gespiegelt in der konfliktuellen Raumerfahrung zwischen Nähe und Abstand. Die Spaltung an sich bedeutet Strukturbildung und somit einen ersten defensiven Mechanismus gegen die Bedrohung des Amorphen. Wie nun «handhabt» Brecht - genauer der epische Stückeschreiber diese Struktur?

5 Eine erste Verschiebung ist grundlegend hinsichtlich des Schauplatzes der Konfliktaustragung: die Projizierung der traumatischen Primärphantasien auf den Bereich des Gesellschaftlichen. Diese Veräußerlichung bedeutet den Versuch, das Subjekt als gesellschaftlichen Funktionsträger in den - dialektischen - Griff zu bekommen. Die Subjektproblematik «aufgehoben» im Kollektiv:

In den wachsenden Kollektiven erfolgt die Zertrümmerung der Person. [...] Sie geht über in anderes, sie ist namenlos, sie hat kein Antlitz mehr, sie flieht aus ihrer Ausdehnung in ihre kleinste Größe - aus ihrer Entbehrlichkeit in das Nichts -; aber in ihrer kleinsten Größe erkennt sie tiefatmend übergegangen ihre neue und eigentliche Unentbehrlichkeit ${ }^{10}$.

6 Besteht doch der unbestreitbare Vorteil der Dialektik darin, Widersprüchliches praktikabel zu machen; auf diese Weise eingefügt, gewinnt das Individuum «einige Sicherheit und ist kalkulierbar» ${ }^{11}$. Es kann zum Einverständnis kommen mit der «Auslöschung»:

Dann seid ihr nicht mehr ihr selber, du nicht mehr Karl Schmitt aus Berlin [...], sondern allesamt ohne Namen und Mutter, leere Blätter, auf welche die Revolution ihre Anweisung schreibt. [...] Dann seid ihr von dieser Stunde an nicht mehr niemand $[. . .]^{12}$.

7 Diese Verschiebung vom Privaten zum Gesellschaftlichen ist an sich kein neuer Befund. Was uns hier aber im Besonderen interessiert, ist aufzuzeigen, in welcher 
Wechselbeziehung privater und gesellschaftlicher Schauplatz stehen, dank welcher Mittel die Bühne des privaten Psychodramas zur epischen Bühne eingerichtet wird, welche latenten Fäden zwischen den Dramaturgien des Privattheaters und der epischen Darstellung gesellschaftlicher Zustände hinund herlaufen.

Alles übergab ich dem Staunen

Selbst das Vertrauteste

Daß die Mutter dem Kinde die Brust reichte ${ }^{13}$.

8 Das Vertrauteste dem Staunen übergeben: dem Intimsten, nennbar gemacht im Urbild der nährenden Mutter, das Vertraute nehmen, es abrücken, es zum Betrachtungsobjekt machen, um die Faszination zu beherrschen. Es auf der epischen Bühne ausstellen, die ja - wie Walter Benjamin Brechts Bühnenrevolution beschreibt - vom Ort der Faszination zum «Podium» werden soll ${ }^{14}$, vom «Bannraum» zu einem «günstig gelegenen Ausstellungsraum $»^{15}$. Die epische Bühne als günstiger Ort, wo das Psychodrama im freudschen Sinn «agiert» werden kann, verstellt gespielt und durchgespielt. Diese latente Beziehung zwischen «Familiendrama» und epischer Demonstration scheint sich in folgender Anmerkung des Stückeschreibers zu verraten. Kommentiert wird die musterhafte Darstellung einer Mutterfigur, die Darstellung der Mutter Wlassowa durch die Weigel:

Sie täuschte nicht nur nicht vor, in Wirklichkeit die Wlassowa zu sein oder sich dafür zu halten, und diese Sätze in Wirklichkeit zu sagen, sondern sie verhinderte sogar, daß der Zuschauer aus Nachlässigkeit und alter Gewohnheit, sich in eine bestimmte Stube versetzte und sich für den unsichtbaren Augenzeugen und Belauscher einer einmaligen intimen Szene hielte ${ }^{16}$.

Bis in den Wortlaut läßt diese Bemerkung das Szenarium einer kindlichen Urszene auftauchen: heimlicher Voyeur und Lauscher, nicht an einem beliebigen, sondern einem «bestimmten» Ort; singularer Zuschauer einer «intimen» Szene, deren erklärte Einmaligkeit das Signum eines Ursprungsphantasmas trägt. Wir lesen diese Bemerkung als das in Form einer Verneinung maskierte Eingeständnis der Faszination des Privattheaters; diese Faszination enthüllt sich als uneingestandene Triebkraft des Bemühens um das musterhafte epische Schauspiel und um den «neuen» Zuschauer, der - «alte Gewohnheit» überwindend - Haltung und Position des kalt-distanzierten Beobachters einneh- men soll; eines Betrachters, den das Schauspiel kalt läßt, der genüßlich rauchend sich als emanzipiert, das heißt als Mann auszuweisen hat. Es handelt sich übrigens um eine schon früh angekündigte Bewältigungsstrategie des armen B.B., wobei das eingesetzte Mittel selbst, dank seiner unübersehbaren oralen Symbolik, sich als Hinweis auf den latenten Konflikt zu erkennen gibt:

Bei den Erdbeben, die kommen werden, werde ich hoffentlich

Meine Virginia nicht ausgehen lassen durch Bitterkeit

Ich, Bertolt Brecht, in die Asphaltstädte verschlagen

Aus den schwarzen Wäldern in meiner Mutter in früher Zeit ${ }^{17}$.

Betrachten wir nun näher einige theatralische Lösungsversuche im Schutz der Dialektik. Zu deren Lob kommentiert Pietzcker: «So bot die Dialektik mit ihrer sich bewegenden Einheit von aktiven und passiven, von "kalten' und 'warmen' Selbstbildern die Möglichkeit, dem endlosen Wechsel zwischen Größen- und Nichtigkeitsphantasien, gefeierten und verachteten Selbstbildern zuentkommen. Die eigene "Vorläufigkeit" wie die des Objekts haben sich in einen Prozeß verwandelt, in dem Subjekt wie Objekt schon in der Gegenwart als historische erscheinen: aus einer schützenden Distanz, die es dem Subjekt leichter macht, die Spannung zwischen eigenen und fremden, bösen 
und guten Anteilen $\mathrm{zu}$ ertragen, sie nicht $\mathrm{zu}$ isolieren, sondern in ihrem Zusammenhang wahrzunehmen und zu gestalten. ${ }^{18}$ In unserer Betrachtung soll das epische Schauspiel zwar in gewisser Hinsicht als Produkt des Rationalisierungsprozesses aufgefaßt werden, aber keineswegs als dessen «Abfallprodukt». Mit Maurice Regnault sehen wir im epischen Spiel eine außerordentlich gelungene ästhetische Kompromißbildung und diese als hervorragendes Kennzeichen des brechtschen Werks überhaupt: «Elle [l'œuvre de Brecht] est l'un des plus fabuleux, efficaces, conséquents efforts pour transformer le rapport avec soi en rapport au monde, le conflictuel imaginaire en analyseur social, l'aliénation en possibilité altruiste, pour tirer de la verticalité l'horizontalité la plus vaste, la plus savante, la plus rigoureuse, la plus sensible.» ${ }^{19}$

\section{«Die Kinder den Mütterlichen ...»}

11 Über die Frage nach der richtigen oder falschen Mutter läßt der Stückeschreiber im Kaukasischen Kreidekreis in einer Gerichtsszene befinden. Er bewerkstelligt eine zweifache Distanzierung, insofern die epische Bühne sich als Spiel im Spiel des Gerichtszeremonials verdoppelt. Tücke der Distanzierungsdramaturgie: Hinter der Maske als Richterinstanz in Sachen Mutter-Kind-Beziehung tritt das Subjekt mit seinem Ambivalenzkonflikt hervor. Der salomonische Urteilsspruch des Richters Azdak nämlich enthüllt sich als das gelungene janusköpfige Produkt der kreativen Regression:

Nehmt zur Kenntnis die Meinung der Alten:

Daß da gehören soll, was da ist, denen, die für es gut sind, also

Die Kinder den Mütterlichen $[. . .]^{20}$.

Der Spaltungsprozeß ist «in den Griff gebracht» durch die Verschiebung des Mütterlichen vom Biologischen auf das Gesellschaftliche. Die natürliche Mutter erweist sich als die böse; diejenige, die nicht Mutter ist, aber mütterlich fühlt und als nährende und erziehende Instanz im Sinne des kindlichen Glücks handelt, als die gute. Die Figurenkonstellation gehorcht zudem in ihrer Verteilung der Rollen der Klassenkampfproblematik: Die herrschende Klasse (Gouverneursfrau) repräsentiert den pervertierten, illegitimen Anspruch auf den Besitz des Kindes; die proletarische Magd die authentische mütterliche Güte, die ihren Wert dadurch erhält, daß sie zur Grundlage einer gesellschaftlichen Utopie erklärt wird. Vorgeführt wird ein «Rechtsspruch» im Sinne der Etablierung eines neuen Rechts, basierend auf einer neuen Mutterschaft; Etablierung nicht des mütterlichen Rechts, sondern des Rechts der Kinder auf die Mütterlichen ${ }^{21}$. Mit diesem Urteil präsentiert sich der Kaukasische Kreidekreis als "goldene Legende» eines geglückten Rationalisierungsprozesses. Die Spuren seiner Elaborierung aber treten in der Ambivalenz des Textes zutage.

«Salomonisch» nämlich ist Azdaks Urteil auch in dem Sinn, als es gleichzeitig mit dem gesellschaftlichen Entwurf Befriedigung eines Primarphantasmas' darstellt, und zwar indem es die faszinierende imago der unberührten Mutter inszeniert. Der Mutter, die nicht geboren, also nicht empfangen hat, die keinen Mann und damit nicht die «Freuden vorher» gekannt hat, wie sie schon folgender früher Dialog zwischen Mutter und Sohn heraufbeschwor:

MUTTER : [...] Noch nicht eine Freude habe ich an dir gehabt, seit du lebst!

BAAL : Aber vorher, Mutter!

MUTTER weint : O du Gotteslästerer. Das ist es! und wie hab ich dafür gebüß...22 

sie verführerisch: Bei den Weiden unten am Fluß, wäscht sie nicht nur das Linnen, an manch heißem Sommertag steckte sie «die Fußzehen und mehr» ins kühle Wasser ${ }^{23}$, das Begehren des Soldaten erweckend. Und zum guten Schluß wird sie ihn ja auch bekommen, ihren Liebsten. Als Mutter aber muß sie als die Unberührte erscheinen. Unermüdlich evoziert der Text, in den verschiedensten Varianten, das Bild der jungfräulichen Mutter, die - im Gegensatz zur falschen leiblichen Mutter ${ }^{24}$ - das Kind «nicht in Freude empfangen» hat ${ }^{25}$; die an einem gewissen Ostermorgen zu dem Kind gekommen ist «wie der Birnbaum zu den Spatzen»" ${ }^{26}$, Schutz und Nahrung gewährend unter der Gefahr selbst "gefressen» zu werden. Sogar das Bild der jungfräulichen Mutter, die dem Kind die Brust reicht, wird entworfen: «Es ist nichts drin, aber du meinst, du trinkst, und das ist etwas.» ${ }^{27}$ Auch wenn diese Geste motiviert ist durch die Härte der Umstände, als Verzweiflungsgeste angesichts der gesellschaftlichen Kälte, ist das Bild da, bedeutet für sich. Ist dem Staunen übergeben und gleichzeitig faszinierend.

Ein anderes - diesmal wenn nicht tragisches, so doch «unglückliches» - Wunsch-Bild der Mütterlichen, die nicht Mutter ist: die stumme Kattrin, «mütterliche» Tochter der Mutter Courage. Ihr Bild konstituiert sich dank einer Serie von Spiegelungen und Umkehrungen, theatralisch veranschaulicht in der Figurenkonstellation. Lebt Mutter Courage vom Krieg, dem sie die eigenen Kinder opfert, stirbt Kattrin durch den Krieg, indem sie die fremden Kinder der Stadt rettet. So wird der Sohn erschossen, weil die Händlerin zu lange gehandelt hat (3. Szene); Beredtheit geht zusammen mit Blindheit, der mütterlichen Unfähigkeit den Sohn vor dem Tod zu schützen. Der von der Weigel dargestellte lautlose Schrei der Mutter an der Bahre des Toten ${ }^{28}$ ist gestische Verdichtung, dank derer hinter dem Bild der kalten Mutter, die ihren dem KriegsHandel geopferten Sohn verleugnet, das Bild der liebenden aufblitzt, die um den Sohn leidet, mater dolorosa. Auf einem Sprachwunder - «Der Stein hat beschlossen zu reden $»^{29}$ - gründet die "gute» mütterliche Aktion der stummen Kattrin, wie Grusche Vorbild wahrer, das heißt auch hier, sozialer Mutterschaft. Auch sie «vorgestellt» als mater immaculata mit der dazugehörigen Ambivalenz: Kattrin, die fasziniert von den roten Stöckelschuhen der Yvette, hinter dem Rücken der Mutter die Hure imitiert; Kattrin, der die Mutter das Gesicht mit Asche bedeckt, damit sie ist «wie ein Stein in Dalarne, wos nix andres gibt, so daß die Leut sagen: den Krüppel sieht man gar nicht» ${ }^{30}-$ zwei Repräsentationen, die ihre Möglichkeiten abstecken zwischen Hure und Jungfrau. Beschädigt, verunstaltet, wird sie schließlich - dem Wunschbild der «reinen» Mutter entsprechend - diejenige sein, auf die kein Mann mehr wartet, auch nicht im Frieden.

Die «revolutionäre» Mutter: eine weitere Variante des dialektischen Umgangs mit dem ambivalenten Mutterkonflikt, ausgestellt auf der epischen Bühne:

So seht ihr also die proletartische Mutter den Weg gehn

Langen gewundenen Weg ihrer Klasse.... ${ }^{31}$

Der «gewundene Weg» gibt sich wieder in seiner doppelten Funktion als gelungene Kompromißbildung $\mathrm{zu}$ erkennen. Auch er führt zur Gründung einer neuen Mutterschaft, genauer er bedeutet die Transfiguration der alten «natürlichen» in eine neue gesellschaftliche. Nach dem Verlust des Sohns durch die Revolution erscheint die Wlassowa mythisiert, zur Mutter der Revolution erhoben:

\footnotetext{
[...] Immer noch Mutter

Mehr noch Mutter jetzt, vieller Gefallenen Mutter

Kämpfender Mutter, Ungeborener Mutter... ${ }^{32}$
} 

Funktion. Gleichzeitig aber - wie die Adoptivmütter Grusche, Kattrin - auch «absolute» Mutter, das heißt Negation des Vaters.

In dieser Hinsicht liest sich auch der "gewundene Weg der proletarischen Mutter» als Demonstration jenes Projekts des epischen Stückeschreibers, «wie die Mutter dem Kind die Brust reichte, dem Staunen [zu] übergeben», das faszinierende Bild der Symbiose von nährender Mutter und Kind in den Griff zu bekommen, gesellchaftlich praktikabel zu machen. Am Beispiel des Nahrungsmotivs zeigt Brecht, wie die Mutter zu «ganz neuartiger, eingreifender Mutterliebe» gelangt, anstelle der «alten abgelebten, familiären $\aleph^{33}$. Letztere manifestierte sich in den Bemühungen der Mutter um die Nahrung für den Sohn, erschöpfte sich in der Sorge darum, dem Sohn eine bessere Suppe herzuschaffen: «das ist Kampf genug für mich $»^{34}$. Der Kampf für den Sohn aber wird sich in einen Kampf mit dem Sohn für alle Söhne verwandeln. Der aus sibirischer Gefangenschaft geflüchtete Sohn kehrt heim zur Mutter. Mit der Druckerei von revolutionären Flugblättern beschäftigt, hat sie keine Zeit, dem Sohn das Brot abzuschneiden:

Kümmert sie sic hum ihn? Keineswegs! Setzt sie ihm Tee vor? Bereitet sie ihm ein Bad? Schlachtet sie ein Kalb? Keineswegs. [...] seine Mutter, statt ihm über das haar zu streiche, nimmt die Blätter heraus ${ }^{35}$.

Im Augenblick der Trennung wird der Hinweis auf die Utopie besserer Zeiten hörbar «Hoffentlich kann ich dir das nächste Mal das Brot abschneiden.» ${ }^{36}$

Mit der faktischen Trennung, die sich als endgültige erweisen wird, ist das Wunschbild der Vereinigung von Mutter und Sohn jedoch keineswegs aufgegeben, sondern aufgehoben in der Idee des gemeinsamen Kampfes um die «dritte Sache».

Er und ich waren zwei, aber die dritte

Gemeinsame Sache, gemeinsam betriebe, war es, die

Uns einte ${ }^{37}$

Lob der dritten Sache: Sie hält die Nähe auf Distanz, die Güte erscheint frei von der Bedrohung der Vereinnahmung:

[...] Wie nahe waren wir uns, dieser Sache

Nahe! ie gut waren wir uns, dieser

Guten Sache nahe! 38

Die epische Darstellung der proletarischen Mutter bedeutet wiederum äußerste Fruchtbarmachung des imaginaren Konflikts für die Konstitution des Subjekts und dessen Sozialisierungsder Welt im Sinne eines gesellschaftlichen Entwurfs.

\section{Die «furchtbare Wahrheit»}

Gemeint ist die des Guten Menschen von Sezuan. Vorgeführt wiederum in einer Szene im Gerichtslokal, diesmal aber nicht Ort eines Urteilsspruchs, wo man die «gute» Wahrheit verkündet, sondern Schauplatz eines Geständnisses, genauer der Enthüllung einer «furchtbaren Wahrheit», demonstriert als Enthüllungsszene im konkreten Wortsinn:

SHUI TA: Dann laßt euch die furchtbare Wahrheit gestehn: ich bin euer guter Mensch!

Er nimmt die Maske ab und reiß sich die Kleider weg, Shen Te steht da.

DER ZWEITE GOTT : Shen Te!

SHEN TE: J, ich bin es. Shui Ta und Shen Te, ich bin beides ${ }^{39}$. 

Shen Te. Dem Furchtbaren diéser Wahrheit Rechnung tragen, heißt Shen Te beim Wort und ernst nehmen: «ich bin beides»; heißt hinter dem Spektakel des komödienhaften Rollentausches die Demaskierung eines ungelösten (unlösbaren?) Konflikts entdecken. Wovor so manche Brechtinterpreten warnen, habe doch Rollenspiel und Rollentausch für den epischen Stückeschreiber nichts zu tun mit identitärem Konflikt, hat er doch vornehmlich in Mann ist Mann - die Demonstration dafür geliefert, daß man eines schönen Morgens sein Ich gegen ein anderes ohne weiteres umtauschen kann. Natürlich ist die Doppelrolle zunächst deutbar als «Ausdruck der Existenzspaltung des Menschen in kapitalistischer Gesellschaft» ${ }^{40}$, Spaltung, die in der optimistischen Perspektive der Veränderbarkeit der Verhältnisse allerdings Konturen des Komischen annehmen kann. Dies aber gilt für die eine Ebene, die des epischen Projekts. Geht dieses aber hier restlos auf? Ist die Parabel etwa klüger als ihr Autor (H. Müller)?

Der Rollentausch scheint uns hier weder der Projektion jenes Ich zu entsprechen, das lustvoll - sich dabei aufs Spiel setzend - mit seinen Masken spielt, noch der Strategie des epischen Spielleiters, der Spaltung und Auflösung des Ich in praktikable Distanz rückt. Das Bewußtsein der Zerrissenheit lastet über dem spielerischen Umgang des Problems, die dialektische Lösungs- und Aufhebungsdynamik verknotet sich zum Paradox, und man kann sich die Frage stellen, ob dem Stückeschreiber hier das epische Spiel mit der Mutterimago nicht in gewisser Hinsicht entgleitet. Notiert er doch selber, gerade in Bezug auf dieses Stück: «interessant, wie sich bei diesen dünnen Stahlkonstruktionen jeder kleinste rechenfehler rächt» ${ }^{41}$. Die Figur der Shen Te ist nicht mehr «aufgehoben» in einer gelungenen Kompromißbildung, sondern zu der schwindelerregenden Projektionsfläche widersprüchlicher imagines geworden.

Als Frau ist sie Prostituierte und Liebende, eine für Brecht ungewöhnliche Konstellation. Ist doch die Prostituierte für ihn Modellfall für die Ausbeutung im kapitalistischen System, wo ihre Arbeitskraft, die Liebesfähigkeit also, nach den Gesetzen dieses selben Systems, notwendiger Weise abgenutzt und erschöpft wird ${ }^{42}$. Shen Te's Handel aber mit der "Ware Liebe» ${ }^{43}$ macht sie nicht unfähig für «wahre Liebe». Sie ist auch Liebende, nicht nur sich aufopfernde, selbstlos Liebende, wie sie um dem vertrauten Schema des gespaltenen Frauenbildes zu genügen - gern gesehen wird, sondern begehrend Liebende. Zwar beginnt ihr Liebeserlebnis strukturgetreu als Rettungsphantasie angesichts des verhinderten, am Leben verzweifelnden Fliegers; der Wunsch dem Flieger zum Fliegen zu verhelfen durchzieht ihre Liebesgeschichte und macht Shen Te zum guten Gegenbild der «bösen» leiblichen Mutter, die dem Sohn die Flügel beschneidet.

Aber Gut-Sein für die andern verdoppelt sich bei Shen Te mit dem Anspruch auf GutSein sich selber gegenüber: «Jeden mit Glück erfüllen, auch sich, das ist gut.» ${ }^{44}$ Nach der Liebesnacht erscheint die erwachende Stadt wie verwandelt, Nicht nur die Zeitungsverkäufer erecken ihr Interesse und «die Mänenr, die den Asphalt mit Wasser überspülen, und den Ochsenkarren mit dem frische Gemüse vom Land $»^{45}$, sie findet Vergnügen an sich selber, betrachtet sich im Spiegel der Schaufenster. Aux Lust, dem Geliebten zu gefallen, bekennt sie sich zur «Leichtsinnigkeit»: Auch wenn es ein paar Schalen reis weniger bedeutet für die hungernden Kinder der Stadt, jauft sie den Schal, um schön zu sein für Sun. Trägt Shen Te's jubilierendes Bekenntnis zur Liebe nicht - in brechtscher Perspektive gesehen - den Akzent des Unerhörten?

Germanica, 18 | 1996 
Ich will mit dem gehen, den ich liebe.

Ich will nicht ausrechnen, was es kostet.

Ich will nicht nachdenken, ob es gut ist.

Ich will nicht wissen, ob er mich liebt.

Ich will mit ihm gehen, den ich liebe ${ }^{46}$.

Aks Publikumsadresse angelegt, erscheinen uns diese Verse eine doppelte Auslegung zu erlauben. Im Sinne der epischen Demonstration der Parabel vom guten Menschen von Sezuan sind sie Verweis auf die Blindheit der Shen Te angesichts der Beschaffenheit der Welt; spätestens anläßlich der Hochzeitsfeier, die ihre Liebe krönen sollte, werden ihr die Augen geöffnet. Aber darüber hinaus sind sie Ausdruck eines Bekenntnisses zu ganz individuellem Liebesglück jenseits der gesellschaflichen Ansprüche und deshalb unerhört.

Wenn Shen Te auf dieses Glück verzichtet, dann letztendlich für den Sohn, den sie erwartet. In keinem anderen Stück Brechts, so scheint es uns, entfaltet sich das Spiel mit der Mutterimago mit solcher in unauflösliche Widersprüchlichkeit verstrickten Komplexität, was uns die Vermutung nahelegt, daß die Dynamik des Primärkonfliktes ungewöhnlich überhand nimmt. Die reisverteilende Shen Te, Vorbild der mütterlichten Güte, «Engel der Vorstädte», wird, um den eigenen Sohn zu retten die soziale Mutterschaft verleugnen. Auf das «graue Mäulchen» des hungrigen Kindes zeigend, das im Abfalleimer nach Nahrung sucht, verkündet sie die «furchtbare Wahrheit»:

Was ich gelernt in der Gosse, meiner Schule

Durch Faustschlag und Betrug, jetzt

Soll est dir dienen, Sohn, zu dir

Will ich gut sein und Tiger une wildes tier

$\mathrm{Zu}$ allen andern, well's sein muß [...] (1573)

Da ist kein weiser Richter, der ihr mit seinem salomonischen Urteilsspruch zur Seite steht, über gute und schlechte Mutterschaft das «schöne» Urteil spricht. Shen Te ist auf sich gestellt in ihrer Zerrissenheit, die sich als Umkehrung der Grusches erweist, hieß es doch dort: «Zerreißen mußte ich mich für das, was nicht mein war/Das Fremde [...]»"

Läßt sich Shen Te's emphatisch-leidenschaftliches Bekenntnis zur «Frucht ihres Leibes», die nur ein Sohn sein kann, noch mit einem gesellschaftlichen Projekt verbinden? Es ist schwierig, ein solches am Horizont des Stückes auszumachen. Wo ist das Kollektiv, in deren Mitte Mutter und Sohn als gesellschaftliche Funktionsträger zu «praktikablen Abbildungen» gelangen können? Wo die «dritte» Sache, um deren Kampf die Vereinigung von Mutter und Sohn vorstellbar werden kann? Anstelle solcherlei Rationalisierungsversuche die Evokation einer lustvollen Intimität von Mutter-Sohn: Mit dem Sohn Hand in Hand auf einem Spaziergang erfüllt sie die mütterliche Rolle der Lehrenden («Komm, Sohn, betrachte dir die Welt.»), sorgt für erquickende Nahrung («Bitte ein Glas frisches Wasser für meinen Sohn.» ${ }^{48}$ ). Es bleibt aber nicht bei der Inszenierung dieser uns aus der epischen Demonstration bekannten mütterlichen Funktionen. Die Expedition artet aus in verbotene Spiele:

Ach der Polizist ! Da machen wir einen Bogen. Vielleicht holen wir uns ein paar Kirschen dort, im Garten des reichen Herrn Feh Pung. Da heißt es nicht gesehen werden. Komm Vaterloser! Auch du willst Kirschen! Sachte, sachte, Sohn! Sie gehen vorsichtig sichumblickend: Nein, hier herum, da verbirgt uns das Gesträuch. Nein, so gradlos drauf zu, das kannst du nicht machen, in diesem Fall. Er scheint sie wegzuziehen, sie widerstrebt. Wir müssen vernünftig sein. Plötzlich gibt sie nach. Schön, wenn du nur gradezu drauflosgehen willst... Sie hebt ihn hoch. Kannst du die Kirschen erreichen? Schieb in den Mund, dort sind sie gut aufgehoben. Sie verspeist 
selber eine, die er ihr in den Mund steckt. Schmeckt fein. Zum Teufel, der Polizist. Jetzt heißt es laufen. Sie fliehen. [... $]^{49}$ glücklicher und geglückter kaum vorstellbar. In ihr verdichten sich Wunschvorstellungen verschiedener Art, genau- er, sie werden durchgespielt: Auf der epischen Bühne öffnet sich als Bühne auf der Bühne die imaginäre des Tagtraums. So folgt der Inszenierung der fürsorglich-führenden mütterlichen Hand, als Gegenbewegung gleichsam, die Phantasie der Transgression. Nicht auf den geraden Wegen von Ordnung und Gesetz bewegt sich die mütterliche Führerin, sondern sie wird zur Verführerin auf Abwege. Die Apostrophe «Vaterloser» versichert die Negation der stören- den, Lust verbietenden väterlichen Instanz, in Aktion umgesetzt in der lustvollen Überlistung des Ordnungshüters und des reichen Besitzers Feh Pun. Um Lust aber noch in einem anderen Sinn geht es hier: Im gemeinsamen Genießen der verbotenen Früchte enthüllt sich das Spiel als erotisches. Signifikante Umkehrung des Nahrungsmotivs, geht dieses doch in der epischen Darstellung gerade mit der entsexualisierten Mutterfunktion zusammen. Wie geschickt inszeniert ist doch die Rollenverteilung in diesem verbotenen Spiel: Der Sohn nicht nur passiv Verführter, übernimmt selbst den Part des Verführers, es gelingt ihm, die Widerstrebende zum Nachgeben zu bewegen; ja mehr noch, er selbst pflückt - wenn auch mit mütterlicher Hilfestellung - die Früchte und steckt sie ihr in den Mund.

Shen Te's Verzicht auf ihre Liebe zu dem verhinderten Flieger Sun wird in höchstem Maß und in verschiedener Hinsicht kompensiert. Der Sohn wird zu ihrem Flieger, ihr «neuer Eroberer» ${ }^{50}$. Ihre Liebeserklärung gibt sich natürlich maskiert, verschoben auf die gesellschaftliche Bühne («Ein Flieger [...], der die Post von Mensch zu Mensch/Über die unwegsamen Wüsten bringt.. $\rangle^{51}$, auf der diesmal aber ungewohnt messianische Akzente zu vernehmen sind:

Man sieht noch nichts. Er ist aber schon da. Die Welt erwartet ihn im Geheimen. In den Städten heißt es schon: Jetzt kommt einer, mit dem man rechnen muß $\Re^{52}$.

Der Schlußszene des Guten Menschen von Sezuan, Parodie einer Gerichtsszene, kann nach Maurice Regnault eine über ihre Funktion in dieser besonderen Fabel hinausgehende Bedeutung abgewonnen werden. Die Enthüllung des Double Shui Ta/Shen Te - vor einer defekten Richterinstanz und also kaum für diese bestimmt - ließe sich als Bild einer poetologischen Enthüllung interpretieren. Unter dem Kostüm des dickleibigen Shui Ta, des kalten Dialektikers, kommt die schwangere Shen Te zum Vorschein, Wunschbild der einzig dem Wohl des Sohnes ergebenen Mutter, bildlich-szenisches Geständnis der versteckten Wahrheit, der geheimen Triebfeder, aller Inszenierungen Selbstinzenierungen - Brechts: «c'est le dévoilement de ce qui est le vrai pour tout le théâtre de Brecht, le vrai de toute une poétique, et l'apparition sous Shui Ta de Shen Te enceinte est vrai- ment l'image de l'aveu, où se dit ce qui en toute œuvre brechtienne est à l'œuvre, où se réalise en toute "naïveté" l'imaginaire brechtien originel. ${ }^{53} \mathrm{Nichts}$ anderes enthüllt ja der Stückeschreiber in der Formulierung seines Projekts, von dem unsere Betrachtungen ausgegangen sind, nämlich selbst das Vertrauteste dem Staunen übergeben zu wollen, und dieses sei letztendlich «daß die Mutter dem Kind [wir präzisieren: dem Sohn] die Brust reichte». 


\section{NOTES}

1. Brechts Werke werden zitiert nach: Bertolt Brecht, Gesammelte Werke (werkausgabe edition suhrkamp), Frankfurt 1967. Hier Bd. XX, S.1062.

2. Die geradezu obsessionelle Art, wie Brechts Texte auf der Repräsentation der mütterlichen Figur - und korrelativ dazu auf der Verdrängung der väterlichen - insistieren, ist von der Kritik allerseits bemerkt worden. Die Liste ist lang von der unverheirateten werdenden Mutter (Sophie in Baal, Anna in Trommeln in der Nacht, Shen Te im Guten Menchen zu Sezuan) über die alleinstehende Mutter (die Mutter Baals, Mutter Courage, Mutter Carrar, Mutter Pelagea Wlassowa, die Mutter des Ja-sagers und Neinsagers, oder die «Adoptiv-Mutter» (Grusche im Kaukasischen Kreidekreis, Kattrin in Mutter Courage und ihre Kinder).

3. Brecht, Der Messingkauf, Bd. XVI, S. 531.

4. Der Herr Puntila und sein Knecht Matti, Bd. IV, 1694.

5. Vom armen B.B., Bd. VIII, S. 261.

6. Cf. Hans-Thies Lehmann, «Das Subjekt der Hauspostille. Eine Lektüre des Gedichts Vom armen B.B.» In Brecht-Jahrbuch 1980, S. 23.

7. Hier ist vor allem auf die Untersuchung von Carl Pietzcker zu verweisen, der Brechts Werk wie es uns scheinen mag, stellenweise zu systematisierend vorgehend - aus dem Primärkonflikt Mutter-Sohn und speziell der Herzneurose erklären will: «Ich kommandiere mein Herz». Zu Brechts Herzneurose - ein Schlüssel zu seinem Leben und Schreiben, Königshausen 1988.

8. Cf. Peter von Matt, «Brecht und der Kälteschock. Das Trauma der Geburt als Strukturprinzip seines Dramas.» In Das Schicksal der Phantasie, München 1994, S. 297-312. Hier S. 306.

9. Cf. dazu Carl Pietzcker, a.a.O.

10. Brecht, Individuum und Masse, Bd. XX, S. 61.

11. Ibid., S. 62.

12. Brecht, Die Maßnahme, Bd. II, S. 637.

13. Lied des Stücke Schreibers, Bd. VII, Schlußbemerkung, S. 10*.

14. Walter Benjamin, Versuche über Brecht, Frankfurt 1966, S. 30.

15. Ibid. S. 8.

16. Anmerkungen zu Stücken und Aufführungen, Bd. XVII, S. 1039.

17. Vom armen B.B., Bd. VIII, S. 263.

18. Pietzcker, a.a.O., S. $177 f$.

Nicht einsehbar erscheint es uns allerdings, daß Pietzcker kritisch anmerkt, Brecht sei nur beschränkt ein Dialektiker gewesen: «Er hat einen Teil seiner selbst, seinen von Ambivalenzen beherrschten Umgang mit dem Unbewußten, seinen "privaten" Bereich abgespalten und dialektisches Verhalten und Denken nur mit dem Blick auf das entwickelt, was sich draußen befand...». (S. 179) Wie sollte es anders sein? Diese Abspaltung ist doch Voraussetzung für den Rationalisierungsprozeß des dialektischen Vorgehens.

19. M. Regnault, a.a.O., S. 82.

20. Der kaukasische Kreidekreis, Bd. V, S. 2105.

21. Cf. Maurice Regnaut : « Être bon, "gut sein für", c'est être fait pour, c'est être propre à assurer POUR LUI une pleine fonction nourricière, et dans le Cercle de craie où la maternité est fondement d'une nouvelle société, cette bonté, valeur absolue, devient l'aptitude à la production de l'humain même et fonde ainsi le droit nouveau, non plus le droit-de mais le droit-à, non plus le droit de la mère (à l'enfant) mais le droit (de l'enfant) à la mère - et plus exactement le droit des enfants aux "gens maternels", aux "Mütterlichen", le "maternel" devenant l'être fondamental de la société. » («Brecht maternel» In L’Herne. Bertolt Brecht 2, Paris 1982, S. 79). 
22. Brecht: Baal. Drei Fassungen. Hg. Dieter Schmidt, Frankfurt 1966, S.22f. (Zit. in Pietzcker, a.a.O., S.9).

23. Der kaukasische Kreidekreis, Bd. V, S.2012.

24. ANWALT [für die falsche Mutter plädierend): Sie hat es empfangen in den heiligen Ekstasen der Liebe... (Bd. IV, S .2096).

25. Ibid., S. 2053.

26. Ibid., S. 2050.

27. Ibid., S. 2027.

28. Anmerkung des Stückeschreibers aus den Modellbüchern: «Eine mini- male Bewegung drückt aus, daß der Schrei aus dem Mutterschoße kommt. Der Krieg hat über eines der größten aller menschlichen Gefühle gesiegt.» (Zit. In Brechts Mutter Courage und ihre Kinder, hg. von K.D. Müller, Frankfurt 1982, S. 110.

29. Anmerkungen zur Courage, ibid., S. 117.

30. Mutter Courage und ihre Kinder, Bd. 4, S. 1380.

31. Anmerkungen zu Stücken und Aufführungen, Bd. XVII, S. 1053.

32. Ibid., S. 1034.

33. Ibid., S. 1040.

34. Die Mutter, Bd. II, S. 834.

35. Ibid.

36. Ibid.

37. Die Mutter, a.a.O., S. 878.

38. Die Mutter, ibid.

39. Der gute Mensch von Sezuan, Bd. IV, S. 1603.

40. Christian Giese, «Der gute Mensch von Sezuan. Aspekte einer brechtschen Komödie.» In: Materialien zu Brechts «Guter Mensch von Sezuan», hg. von Jan Knopf, Frankfurt 1982, S. 221.

41. Aus dem Arbeitsjournal, zit. in Materialien, a.a.O., S. 13.

42. Cf. Giese, a.a.O., S. $229 f$.

43. Die Formel «Die Ware Liebe» hatte Brecht in einem frühen Entwurf als Titel des Stücks vorgesehen. (Cf. Materialien zu Brechts «Guter Mensch von Sezuan», a.a.O., S. 111).

44. Der gute Mensch von Sezuan, Bd. IV, S. 1532.

45. Ibid., S. 1552.

46. Ibid., S. 1552.

47. Der kaukasische Kreidekreis, Bd. 5, S. 2074. (Herv. LH.)

48. Der gute Mensch von Sezuan, Bd. IV, S. 1568.

49. Ibid.

50. Ibid.

51. Ibid.

52. Ibid.

53. Maurice Regnault, «Brecht maternel», a.a.O., S. 77.

\section{RÉSUMÉS}

Die Mutter-Sohn-Beziehung als rekurrentes Thema des brechtschen Theaters ist geradezu augenfällig. Die Regressionsphantasie soll in ihrer «kreativen Funktion» (Peter von Matt) ins 
Auge gefaßt werden, als Strategie einer produkti- ven Rationalisierung des Primäkonflikts. Die epische Bühne mit den ihr eigenen Verfahren erscheint in dieser Perspektive als der geeignete Ort, wo das Psychodrama «durchgespielt», im freudschen Sinn «agiert» werden kann. Varianten dieses epischen Spiels mit dem ambivalenten Mutterkonflikt: die Repräsentation der sozialen Mutterschaft (Grusche, Kattrin); das Bild der «revolutionären" Mutter/Mutter der Revolution (die Mutter Wlassowa). In dem «guten Menschen» Shen Te scheint sich das dialektische Spiel allerdings zum Paradox zu verknoten.

La relation mère/fils comme thème récurrent du théâtre brechtien est une évidence. Le fantasme régressif est envisagé dans sa «fonction créative " (Peter von Matt), comme stratégie d'une rationalisation productive du conflit primaire. Dans cette perspective, la scène épique avec les procédés qui lui sont propres apparaît comme le lieu privilégié ou le psychodrame peut se donner en spectacle, ou il peut être « acte » («agiert»), au sens freudien du terme. Variantes de ce jeu avec ce conflit maternel ambivalent: la représentation de la maternité sociale (Grusche, Kattrin), l'image de la mère « révolutionnaire » / Mère de la Révolution (la mère Wlassowa). C'est dans la représentation de la «bonne âme » Shen Te que le jeu dialectique se noue pour devenir paradoxe.

\section{AUTEUR}

\section{INGRID HAAG}

Université de Provence 